\title{
Hyperuricemia and cardiovascular implications
}

\author{
KAIVAN PATEL ${ }^{1}$, MICHAEL F. BLACKARD ${ }^{2}$, DIVYA A. PANDYA ${ }^{3}$, \\ PUJA SRIVASTAVA ${ }^{4}$, MANISH GUPTA $^{5}$, ABHIJIT S. PANDYA $^{4}$ \\ ${ }^{1}$ Pacific Medical College and Hospital, Udaipur, India. \\ ${ }^{2}$ B.S., Nova Southeastern University, College of Osteopathic Medicine, 3301 College Avenue, Fort Lauderdale, FL, \\ 33314, USA. \\ ${ }^{3}$ D.O., Broward Health Medical Center, 1600 S Andrews Ave, Fort Lauderdale, FL, 33316, USA. \\ ${ }^{4}$ DM, CIMS Hospital, Ahmedabad, India \\ ${ }^{5}$ Sports Orthopedic Center, 9325 Glades Road, Suite 205, Boca Raton, FL, 33434, USA. \\ ${ }^{6}$ Florida Atlantic University, Department of Computer and Electrical Engineering and Computer Science, 777 Glades \\ Road, EE 516, Boca Raton, FL 33431, USA.
}

\begin{abstract}
Gout is a painful disease that leads to inflammation and joint damage brought on by monosodium urate formation and deposition in joints. Multiple comorbidities are associated with gout, including and not limited to obesity, diabetes, hyperlipidemia, hypertension, vascular diseases and many other cardiovascular diseases. When the symptoms of gout are ignored or appropriate treatment is not prescribed, patients can develop a severe, chronic form of gout called tophaceous gout.
\end{abstract}

Key-Words: - gout, tophaceous, coronary artery disease, CAD, colchicine, hypertension, cardiovascular disease, hyperuricemia, uric acid, monosodium urate crystals, MSU

Received: April 9, 2020. Revised: October 31, 2020. Accepted: November 12, 2020. Published: December 31, 2020.

\section{Introduction}

Gout is commonly viewed by many as a disease of lifestyle choices. However, recent studies suggest that diet contributes minimally to the development of gout versus genetics [1]. Gout is also a component of metabolic syndrome along with high blood pressure, obesity, stress, and diabetes. While gout is typically thought of as a painful but somewhat benign condition by the general population, more and more studies are published indicating otherwise. Gout is defined by the existing or the prior existence of clinical symptoms including painful swelling [2]. Chronically untreated gout can lead to permanent damage to joint structures and can affects other organ systems.

For general understanding, gout is deposition of monosodium urate crystals (MSU) in the joints and soft tissue forming tophi [3]. The most common sites of MSU deposition are 1st metatarsal dorsal cartilage, patellar tendon, 1st metatarsophalangeal (MTP) and least frequently found in the 1st-5th metacarpophalangeal (MCP) on the palmar aspect, posterior aspect of the elbow, deep infrapatellar bursa, gastrocnemius-semimembranosus bursa, and 5th MCP dorsal and palmar cartilage [4].

Undiagnosed or untreated gout can lead to tophaceous gout presenting in patients as painful swelling and possible erosions of the skin and joints [3]. The swelling has regular margins unlike in cases of acute gout in which the margins tend to be more irregular.

The development of gout can be divided into the pre-clinical and the clinical states [2]. In the preclinical stages of gout, one can develop hyperuricemia, MSU crystal deposition, and hyperuricemia with MSU crystal deposition without clinical symptoms. Approximately $20-25 \%$ of hyperuricemic patients have MSU crystal deposits confirmed by synovial fluid analysis, ultrasound, or dual-energy CT (DECT)[5]. The clinical, or symptomatic, states include gout, tophaceous gout, and erosive gout [2]. Historically, gout was known to be a disease of the affluent as they were able to afford meats and alcohol that contribute to the condition [3]. New evidence points to genetics over lifestyle as the major contributor to hyperuricemia [6]. Genome studies have identified three genes SLC2A9, ABCG2, and SLC22A12, in addition to 55 loci among European and Japanese ancestry as the major determinants of uric acid balance with significant correlations to many cardio-metabolic traits. Additional risk factors for gout can be seen in (Figure 1). 


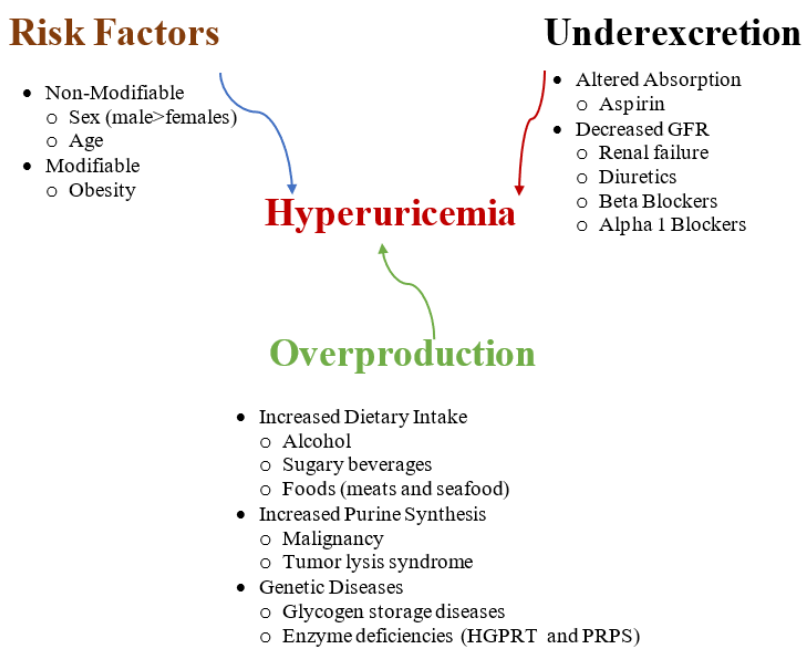

Figure 1. Risk factors for developing hyperuricemia

Gout indicates a hyperuricemic state in the body suggests some imbalance in the body's ability to regulate the intake and excretion of uric acid. This hyperuricemic state can result from increased intake, beyond the body's ability to regulate, or decreased excretion, possibly stemming from a disruption in the ability of the kidney, at either functional or morphological level. Hyperuricemia has been found to be prevalent among patients of coronary artery disease (CAD) which points towards association between uric acid levels and CAD [7].

\section{Pathogenesis:}

The development of gout is based on the derangement of purine metabolism by various etiologies. Purines are used to build DNA and RNA, but during cellular breakdown can be reused or eliminated. When the elimination or salvage pathways are deranged, hyperuricemia can arise. The etiologies can be broken down into either increased production or decreased elimination of uric acid. The increased uric acid in the blood lead to deposition of MSU crystals in joints and the inflammatory response that results orchestrated by macrophages and neutrophils [8]. These crystals themselves are not are not recognized by the immune system [9]. However, due to the hydrophobic surface of the crystal, it can become coated in proteins such as $\operatorname{IgG}$, serum CD14, and other serum proteins that cause the immune system to react. The macrophage's Toll-like receptors interact with CD14 resulting in phagocytosis of the crystal. The recognition of the crystals through the NLRP3 cytosolic pattern recognition receptor (PRR) signals the assembly of an inflammasome leading to the release of inflammatory mediators by the cell, most importantly IL-1 $\square[8]$. Chronically untreated gout can lead to deposition of MSU crystals in the soft tissues surrounding joints causing recurrent inflammation and eventual joint destruction. Gout been associated with other clinical conditions including chronic renal disease, atherosclerotic cardiovascular disease, hypertension, obesity, insulin resistance, and hyperlipidemia, which may occur together as the metabolic syndrome $[10,11]$.

Research has indicated uric acid to be an independent risk factor for MI or CAD [12]. Here we are presenting a case of tophaceous gout which remained undiagnosed for almost eight years.

\section{Case Presentation:}

The patient is a 72-year-old Indian man with a past medical history of ischemic heart disease, untreated hypertension, and coronary artery bypass graft surgery $(\mathrm{CABG})$ for severe triple vessel disease in 2011. A post-surgical 2D ECHO confirmed normalization of all the previous abnormalities with initial improvement in left ventricular ejection fraction (LVEF) of $35 \%$ from $25 \%$ in pre-surgical studies.

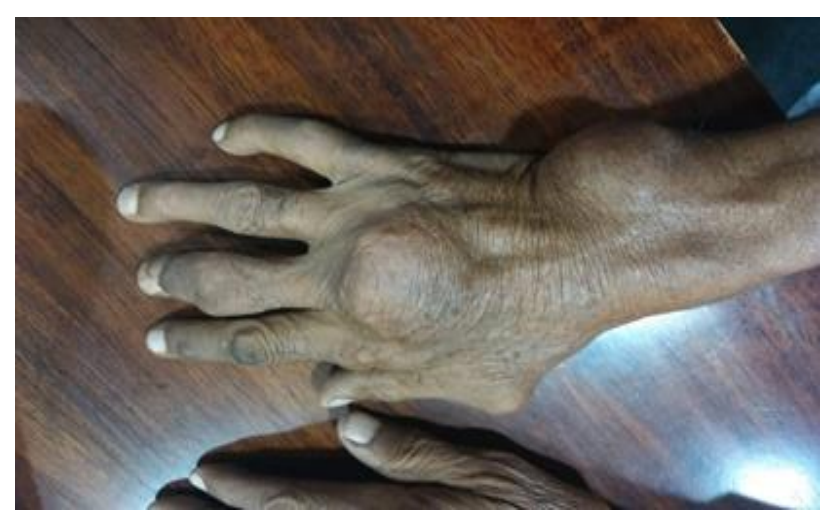

Figure 2. Right hand with tophaceous gout present on first phalanx, third metacarpophalangeal and proximal interphalangeal joints, fifth proximal interphalangeal joint.

The patient came for cardiology checkup in 2019 and during this visit, joint swelling was noticed. On inquiry, it was revealed that he had been suffering with joint swelling primarily on the 1st MTP for approximately the last 6-8 years and was unable to recall whether symptoms were present prior to CABG. The patient denied any prior diagnosis of gout or hyperuricemia and it is unclear if the patient was taking any medications during this period. The initial onset was on the 1st MTP and was followed 
by swelling on the dorsal foot (Figure 3), the wrists bilaterally, right first phalanx, right fifth proximal interphalangeal joint and third MCP and proximal interphalangeal joints (Figure 2).

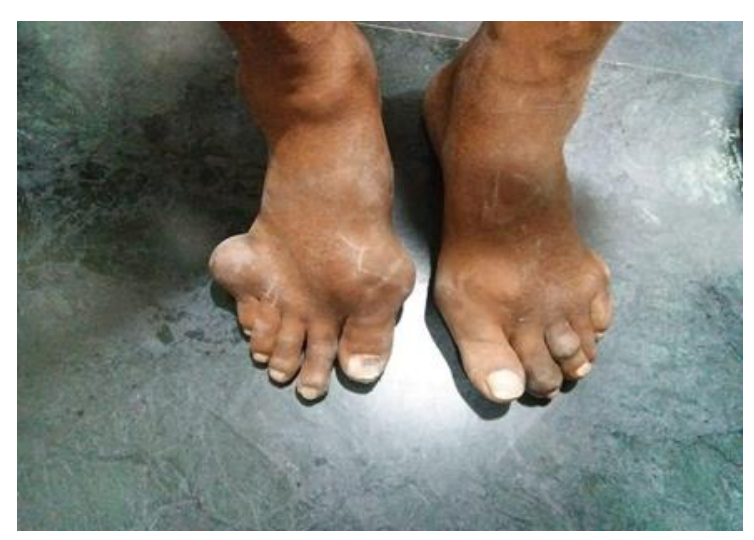

Figure 3. Tophi on right MTP, bilateral dorsal

surface of the foot, fifth MTP, left third

interphalangeal joint.

Laboratory examination revealed uric acid level of $9.6 \mathrm{mg} / \mathrm{dl}$ while all other laboratory parameters were normal. X-ray examination revealed multiple punched out erosions in para-articular portion of all the sites involved (Figure 4). The punched-out lesions have the characteristic sclerotic overhanging edges seen in chronic gout. On examination by the rheumatologist, tophi swelling were confirmed. A diagnosis of gout was made based on this lab, clinical, and historical data and a calculated patient score of 10.5 putting patient at high risk for gout using the diagnostic rule described by Janssens et al., 2010 [13].

In the case of gout patients, joint spaces are preserved until quite late in the disease process. Even if the joint is partially destroyed (usually on the dorsal aspect), preservation of another area of the joint (usually flexor aspect) creates the radiographic impression that the joint space is preserved. This is demonstrated at the PIP joint in the Figure 4, X-rays are critical in distinguishing between gout and rheumatoid arthritis. In gout, mineralization is maintained and there is no predominant periarticular osteopenia. In the X-ray (Fig. 4), it can be noted that despite multiple erosions at the PIP joint, there is no osteopenia. Erosions in gout patients often occur in a very sporadic asymmetric distribution. Due to the indolence of the process borders can be sclerotic, which results in a punched-out or "mouse-bitten" appearance as seen in Figure 4. The edges of the cortex undergo remodeling in an outward fashion as the erosion develops which results in an overhanging edge. The X-ray in Figure 4 (pink arrow) vividly demonstrates this. Lytic lesions can be seen in the X-ray image (yellow arrows in Figure 4). Due to the chronicity of the process, bone production leads to sclerotic borders, irregular spicules at the sites of tendon and ligament attachments overhanging edges and bony enlargement which can be noted in the X-ray.

The patient was then started on gout treatment with febuxostat $40 \mathrm{mg}$, colchicine $0.5 \mathrm{mg}$ and naproxen $500 \mathrm{mg}$ when and if required. The patient was asked to maintain compliance with medications and was expected to follow up regularly with primary care physician and cardiologist.

\section{Discussion:}

Gout is a well described disease stemming from lifestyle, genetics, or comorbidities. Hyperuricemia is not always easy to recognize as it can be many years before initial presentation as an acute gouty attack. Patient non-compliance to therapies or lack of therapy can lead to chronic gout and eventually tophaceous gout where the crystal deposits in the soft tissue surrounding joints activates while inhibiting osteoblasts resulting in bony erosions [17]. More importantly, studies have found that the damage caused by this hyperuricemic state extends far beyond that of the joint and surrounding soft tissue.

Many studies have found a well-established increase in prevalence of comorbidities among gout patient populations. These increased uric acid levels related to gout are associated with a number of cardiovascular conditions such as hypertriglyceridemia, hypertension, heart failure, atrial fibrillation, aortic stenosis, ischemic stroke, peripheral vascular disease, and coronary artery disease (CAD) [14].The proposed mechanism by which gout affects $\mathrm{CAD}$ is by promoting systemic inflammation. Coronary artery disease pathogenesis is based on inflammation leading to plaque buildup which is worsened by the increase in inflammatory mediators caused by MSU [15]. Some studies not only found that cardiovascular disease accounted for up to 50 percent of deaths of patients with gout, but that there was increasing mortality with increasing severity of gout [14].

Diagnosis of an acute gout flare should be made by conducting an arthrocentesis of synovial fluid of a tophus or joint and the visualization of needle-like birefringent crystals seen under polarizing light microscopy [16]. Additionally, asymptomatic hyperuricemia or gout may be diagnosed by visualizing hyperechogenic areas without posterior acoustic shadowing on ultrasonography or specific 
identification of urate deposits by the differential absorption of x-rays on DECT [17].

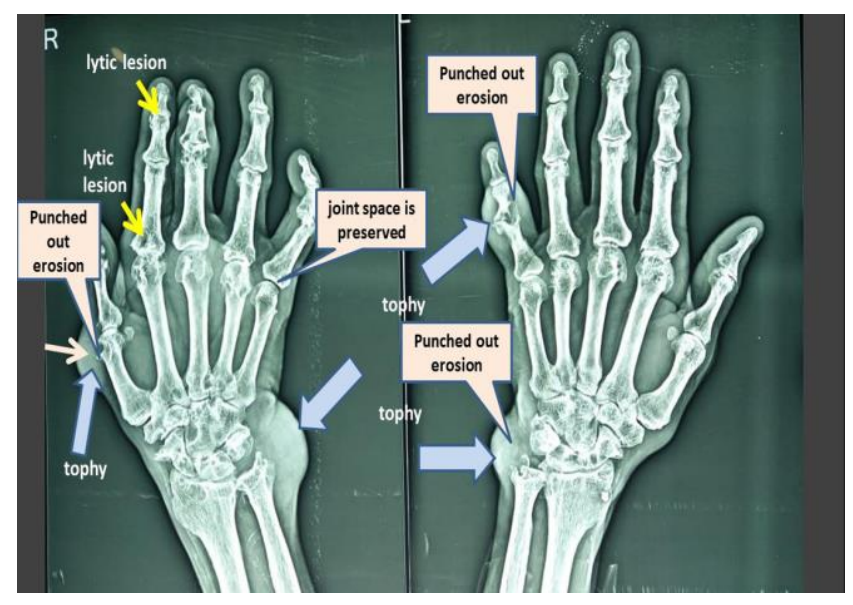

\section{Figure 4. Bilateral hand x-rays}

Under circumstances when laboratory and equipment access are limited, diagnosis of gout can be made by calculating a patient score based on laboratory values, clinical examination, and history. In this diagnostic rule described by Janssens et al., 2010 and validated by Kienhorst et al., 2014, points are awarded in the following manner $[13,18]$ :

Male sex -2 points

Previous patient-reported arthritis flare -2 points

Onset within 1 day -0.5 points

Joint redness -1 point

First MTP joint -2.5 points

Hypertension or at least one cardiovascular disease -1.5 points

Serum urate level $>5.88 \mathrm{mg} / \mathrm{dL}-3.5$ points

With a score of less than or equal to 4 , the patient is considered to be at low risk for gout. Intermediate risk is defined by greater than 4 but less than 8 and high risk is greater than or equal to 8 .

Treatment for gout varies based on the two categories of treatment for prophylaxis or acute attack. For acute attacks, the best medications recommended are NSAIDS, colchicine, or corticosteroids while for prophylaxis, urate lowering therapy is best such as allopurinol, febuxostat, or probenecid. According to recently updated American College of Rheumatology guidelines, a serum urate level of less than $6 \mathrm{mg} / \mathrm{dL}$ should be targeted to dissolve urate crystals and during initial treatment of flares, serum urate levels should be monitored every 2 to 5 weeks. After reaching under $6 \mathrm{mg} / \mathrm{dL}$, urate levels can be measured every 6 months [19].
Allopurinol and colchicine have been further studied to reveal that they may be cardioprotective due to their anti-inflammatory effects. Colchicine is proposed to modulate the production of cytokines and inhibit intimal growth preventing progression of stenosis and restenosis in diabetic patients with stents [15]. Additionally, a recent trial, Colchicine Cardiovascular Outcomes Trial, COLCOT, concluded that colchicine led to a significant reduction in cardiovascular events post myocardial infarction [20]. Furthermore, xanthine oxidase inhibitors, such as allopurinol, have been proposed to have a dual effect on decreasing systemic inflammation by reducing serum uric acid levels and scavenging free radicals [15].

In the treatment of gout in patients with cardiovascular comorbidities, medications must be carefully managed due to the possible effects on uric acid levels. The use of cardioprotective, low-dose, aspirin has been shown to increase serum uric acid levels by increasing reabsorption in the kidneys putting patients at risk of flares [3]. Many antihypertensive agents have been shown to cause an increase in risk of gout such angiotensin converting enzyme (ACE) inhibitors, beta blockers, thiazide and loop diuretics, and angiotensin II receptor blockers (ARBs). Anti-hypertensive medications that are preferred for gout are losartan and calcium channel blockers [21].

Due to the high association between gout and cardiovascular disease and the effects of the inflammatory state caused by hyperuricemia, we recommend patients with cardiovascular conditions be screened for asymptomatic gout. Early diagnosis can inhibit the progression of inflammatory processes by treating the inflammation caused by hyperuricemia and gout. Treatment of gout with allopurinol and colchicine can provide cardioprotection while limiting inflammation resulting from hyperuricemia.

\section{CONCLUSION:}

The inflammatory state induced by hyperuricemia can be linked to the progression of inflammatory diseases and there is evidence that limiting these detrimental effects may be feasible. Addressing treatable reasons for inflammation in the early and possibly asymptomatic stages may limit progression of inflammatory processes such as those in CAD.

Clinical Message:

As the myriad of new research points to a link between hyperuricemia and other conditions including cardiovascular conditions, it may be advantageous to research the practicality, applicability, and potential health benefits that may arise from screening patients, especially those with 
cardiovascular comorbidities, for asymptomatic hyperuricemia.

\section{Consent:}

Informed consent was obtained from the patient to publish case details, test results, and images.

Competing interests:

No competing interests to disclose.

\section{References:}

[1] Major, T. J., Topless, R. K., Dalbeth, N., \& Merriman, T. R. Evaluation of the diet wide contribution to serum urate levels: meta-analysis of population based cohorts. BMJ (Clinical research ed.) 2018; $363, \quad$ k3951. https://doi.org/10.1136/bmj.k3951

[2] Bursill D, Taylor WJ, Terkeltaub R, et al. Gout, Hyperuricaemia and Crystal-Associated Disease Network (G-CAN) consensus statement regarding labels and definitions of disease states of gout. Ann Rheum Dis 2019;78(11):1592-1600. doi:10.1136/annrheumdis-2019-215933

[3] Ragab, G., Elshahaly, M., \& Bardin, T. Gout: An old disease in new perspective - A review. Journal of advanced research 2017; 8(5), 495-511. https://doi.org/10.1016/j.jare.2017.04.008

[4] Perez-Ruiz, F., Dalbeth, N., \& Bardin, T. (2015). A Review of Uric Acid, Crystal Deposition Disease, and Gout. Advances in Therapy 2015; 32(1), 31-41. doi: 10.1007/s12325-014-0175-z

[5] Dalbeth N, Stamp L. Hyperuricaemia and gout: time for a new staging system? Annals of the Rheumatic Diseases 2014; 73:1598-1600.

[6] Tin, A., Marten, J., Halperin Kuhns, V.L. et al. Target genes, variants, tissues and transcriptional pathways influencing human serum urate levels. Nat Genet 2019; 51, 1459-1474. https://doi.org/10.1038/s41588-019-0504-x

[7] Muiesan, M. L., Agabiti-Rosei, C., Paini, A., \& Salvetti, M. Uric Acid and Cardiovascular Disease: An Update. European cardiology 2016; 11(1), 5459. https://doi.org/10.15420/ecr.2016:4:2

[8] So, A., Martinon, F. Inflammation in gout: mechanisms and therapeutic targets. Nat Rev Rheumatol 2017; 13， 639-647. https://doiorg.ezproxylocal.library.nova.edu/10.1038/nrrheum. 2017.155

[9] Wu, M., Tian, Y., Wang, Q. et al. Gout: a disease involved with complicated immunoinflammatory responses: a narrative review. Clin Rheumatol 2020. https://doi.org/10.1007/s10067-020-05090-8

[10] Iseki, K., Ikemiya, Y., Inoue, T., Iseki, C., Kinjo, K., \&Takishita, S. Significance of hyperuricemia as a risk factor for developing ESRD in a screened cohort. American Journal of Kidney Diseases 2004; 44(4), 642-650. doi: 10.1016/s02726386(04)00934-5

[11] Slot, O. Hyperuricemia. UgeskrLaeger 1994; 156(16), 2396-401.

[12] Borghi, C., \& Cicero, A. F. In response: Hyperuricemia as a risk factor for cardiovascular disease. Expert Review of Cardiovascular Therapy 2014; 13(1), 21-22. doi: $10.1586 / 14779072.2015 .987130$

[13] Janssens HJEM, Fransen J, van de Lisdonk EH, van Riel PLCM, van Weel C, Janssen M. A Diagnostic Rule for Acute Gouty Arthritis in Primary Care Without Joint Fluid Analysis. Arch Intern Med. 2010; 170(13):1120-1126. doi:10.1001/archinternmed.2010.196

[14] Perez-Ruiz, F., Martínez-Indart, L., Carmona, L., Herrero-Beites, A. M., Pijoan, J. I., \& Krishnan, E. Tophaceous gout and high level of hyperuricaemia are both associated with increased risk of mortality in patients with gout. Annals of the Rheumatic Diseases 2013; 73(1), 177-182. doi: 10.1136/annrheumdis-2012-202421

[15] Richette, P., Frazier, A., \& Bardin, T. Impact of anti-inflammatory therapies, xanthine oxidase inhibitors and other urate-lowering therapies on cardiovascular diseases in gout. Current Opinion in Rheumatology 2015; 27(2), 170-174. doi: 10.1097/bor.0000000000000149

[16] Vaidya, B., Bhochhibhoya, M., \&Nakarmi, S. Synovial fluid uric acid level aids diagnosis of gout. Biomedical reports 2018; 9(1), 60-64. https://doi.org/10.3892/br.2018.1097

[17] Pascual E, Addadi L, Andrés M, Sivera F. Mechanisms of crystal formation in gout-a structural approach. Nat Rev Rheumatol. 2015; 11(12):725730. doi:10.1038/nrrheum.2015.125

[18] Kienhorst, L. B. E., Janssens, H. J. E. M., Fransen, J., \& Janssen, M. The validation of a diagnostic rule for gout without joint fluid analysis: a prospective study. Rheumatology 2014; 54(4), 609-614.

https://doi.org/10.1093/rheumatology/keu378

[19] Aung, T., Myung, G., \& FitzGerald, J. D. Treatment approaches and adherence to uratelowering therapy for patients with gout. Patient preference and adherence 2017; 11, 795-800. https://doi.org/10.2147/PPA.S97927

[20] Tardif, J.-C., Kouz, S., Waters, D. D., Bertrand, O. F., Diaz, R., Maggioni, A. P., ... Roubille, F. Efficacy and Safety of Low-Dose Colchicine after Myocardial Infarction. New England Journal of Medicine 2019; 381(26), 2497-2505. https://doi.org/10.1056/NEJMoa1912388 
WSEAS TRANSACTIONS on BIOLOGY and BIOMEDICINE DOI: 10.37394/23208.2020.17.16

[21] Choi HK, Soriano LC, Zhang Y, Rodríguez LA. Antihypertensive drugs and risk of incident gout among patients with hypertension: population based case-control study. BMJ. 2012; 344:d8190. Published 2012 Jan 12. doi:10.1136/bmj.d8190

\section{Creative Commons Attribution License 4.0} (Attribution 4.0 International, CC BY 4.0)

This article is published under the terms of the Creative Commons Attribution License 4.0

https://creativecommons.org/licenses/by/4.0/deed.en_US 